\title{
What are the Risk Factors for Non-Alcoholic Fatty Liver Disease after Pancreatoduodenectomy?
}

\author{
Mitsugi Shimoda ${ }^{1}$, Takayuki Shimizu ${ }^{2}$, Yoshimi Iwasaki ${ }^{2}$, Masato Kato ${ }^{2}$, Keiichi Kubota ${ }^{2}$
}

Corresponding author:

Mitsugi Shimoda, M.D., Ph.D.

3-20-1, Chuo, Ami, Ibaraki

300-0395, Japan

Tel: $+81-29-887-1161$

Fax: $+81-29-840-2089$

E-mail: mshimoda@tokyo-med.ac.jp

\section{Abbreviations:}

PD - Pancreatoduodenectomy;

NAFLD - Non-Alcoholic Fatty Liver Disease;

$\mathrm{Fe}$ - Iron;

Zn - Zinc;

$\mathrm{Cu}$ - copper;

SSPPD - Subtotal Stomach-Preserving PD;

HU - Hounsfield Unit;

DGE - Delayed Gastric Emptying;

PF - Pancreatic Fistula;

PJ - Pancreatojejunostomy;

CJ - Choledochojejunostomy;

ROC - Receiver Operating Characteristic
'Department of Gastroenterological Surgery, Tokyo Medical University, Ibaraki Medical Center ${ }^{2}$ Second Department of Surgery, Dokkyo Medical University, Japan

\section{ABSTRACT}

Background: Although hepatic steatosis is often observed after pancreatoduodenectomy (PD), its preoperative clinical features remain unclear. This study focused on the risk factors for non-alcoholic fatty liver disease (NAFLD) after PD.

Methotology: Between April 2008 and June 2011, 100 patients underwent subtotal stomach-preserving PD (SSPPD) for pancreatic head or periampullary diseases. We divided the patients into two groups: a NAFLD group (NA group) and a non-NAFLD group (non-NA group). We compared risk factors such as BMI, blood parameters, blood cell count and trace elements (Fe, Zn, Cu and pre-albumin) in the serum between the two groups. NAFLD was defined as being present when the $\mathrm{HU}$ value was less than 40 or when the spleen/liver HU ratio was more than 1.5 on the basis of abdominal CT three months after surgery.

Results: Twenty-seven of the 100 (27.0\%) patients had NAFLD within three months after surgery. Univariate analysis selected age, reconstruction for Gastrojejunostomy, the preoperative Alb, AST, ALT, Fe, and Zn levels as risk factors for NAFLD. At multivariate analyses, only preoperative Fe level was a risk factor for NAFLD (odd ratio1.052; 95\% C.I.1.45729.663; $p=0.034$ ).

Conclusions: Preoperative serum Fe level is the only independent risk factor for NAFLD after PD. Key words: pancreatoduodenectomy (PD), iron (Fe), zinc (Zn), non-alcoholic fatty liver disease (NAFLD)

\section{INTRODUCTION}

Pancreaticoduodenectomy (PD) is a standard surgical treatment for patients with pancreatic or periampullary tumors. In the last decade, PD has become a safely performed procedure in high-volume surgical institutions. With improvements in operative techniques, high-quality perioperative management, and advances in modalities for patient selection, the postoperative mortality rate has decreased to less than $5 \%(1,2)$. However, during the postoperative course, a proportion of patients show poor food intake or weight loss, and some develop non-alcoholic fatty liver disease (NAFLD). Previous reports have indicated that NAFLD occurs in 23-37\% of patients after PD, and that its risk factors include the volume of resected pancreas, postoperative diarrhoea, lack of hyperlipidaemia, 
and insulin resistance $(3,4)$. On the other hand, some previous studies have suggested that trace elements such as iron ( $\mathrm{Fe})$, zinc $(\mathrm{Zn})$ and copper $(\mathrm{Cu})$ might facilitate the development of $\operatorname{NAFLD}(5,6)$. However, the role of serum trace elements in the development of NAFLD after PD has remained unclear.

In this retrospective study of 100 consecutive PDs, we evaluated the influence of pre- and postoperative factors, operative technique, and commonly accepted risk factors on the incidence of NAFLD, focusing particularly on trace elements in the serum such as iron (Fe), zinc (Zn), copper (Cu), and prealbumin.

\section{MATERIAL AND METHOD}

\section{Patients}

Patients who underwent subtotal stomach-preserving PD (SSPPD) for pancreatic head and periampullary tumors at our hospital between April 2008 and June 2011 were evaluated in this study. One patient who had NAFLD before surgery, and two with a history of alcohol abuse were excluded. A total of 100 patients were enrolled. All clinical, pathological and biochemical data were collected, and analyzed retrospectively. Informed consent was obtained from all of 100 patients in this retrospective study. In our analysis, we compared data between the NAFLD group (NA group) and non-NAFLD group (non-NA group).

\section{Preoperative evaluation}

Preoperative evaluations included physical examination, routine laboratory tests, and contrast-enhanced computed tomography (CT). To be considered for resection, patients had to fulfil the following objective radiographic criteria: 1) absence of metastatic lesions; 2) no evidence of tumour involvement of the superior mesenteric artery or celiac axis. Biliary drainage was performed in patients with jaundice via the percutaneous trans-hepatic or naso-biliary route (PTBD or ENBD) in order to reduce the serum bilirubin level to less than $5 \mathrm{~g} / \mathrm{dl}$.

\section{Postoperative management}

For at least 7-10 days after surgery, all patients received total parenteral nutrition (TPN) along with a trace element solution at $2 \mathrm{ml} /$ day (Fe: $4.73 \mathrm{mg} / \mathrm{ml}$, Zn: $8.625 \mathrm{mg} / \mathrm{ml}, \mathrm{Cu}: 0.624 \mathrm{mg} / \mathrm{ml}$, manganese (Mn): $0.09895 \mathrm{mg} / \mathrm{ml}$ ) and a proton pump inhibitor (PPI) or histamine $\mathrm{H}_{2}$-receptor antagonist. The nasogastric tube (NGT) was left in place until postoperative day (POD) 5 to 7. We performed an upper $\mathrm{Gl}$ series in all cases. If the patients did not show leakage or stenosis of the anasto- mosis, oral intake was started with water or tea and progressed in multiple steps every 1-2 days to a solid diet. However, the NGT was reinserted in cases of vomiting or nausea. Pancreatic enzymes (pancreatin 3 g/day: Yoshida Pharmaceutical Co. Ltd, Tokyo, Japan) were given to all patients and continued for 1 year after surgery.

\section{Blood samples}

Blood samples were obtained preoperatively (Pre), on POD 7 and 21, and 3 months after the operation, respectively. Blood chemical measurements included total protein (TP, g/dl), albumin (Alb, g/dl), prealbumin (Pre-Alb, mg/dl), aspartate aminotransferase (AST, IU/L), alanine aminotransferase (ALT, IU/L), $\gamma$-glutamyl transpeptidase ( $\gamma$-GTP, IU/I), alkaline phosphatase (ALP, $\mathrm{IU} / \mathrm{l})$, blood urea nitrogen (BUN, $\mathrm{mg} / \mathrm{dl})$, creatinine $(\mathrm{Cr}$, $\mathrm{mg} / \mathrm{dl})$, blood sugar ( $\mathrm{BS}, \mathrm{g} / \mathrm{dl})$, electrolytes ( $\mathrm{Na}, \mathrm{K}$ and $\mathrm{Cl}$, $\mathrm{mEq} / \mathrm{l}$ ), blood cell counts (WBC: $\times 10^{3} / \mu \mathrm{l}, \mathrm{RBC}: \times 10^{6} / \mu \mathrm{l}$, hemoglobin: $\mathrm{Hb}, \mathrm{g} / \mathrm{dl}$, platelets: Plt, $\mathrm{x} 10^{3} / \mu \mathrm{l}$, neutrophils: $\mathrm{Neu}, \%)$, trace elements ( $\mathrm{Fe}, \mathrm{Zn}, \mathrm{Cu}: \mu \mathrm{g} / \mathrm{dl})$, and $\mathrm{C}$-reactive protein (CRP, $\mathrm{mg} / \mathrm{dl}$ ).

\section{Definition of postoperative complications}

NAFLD was defined on the basis of a median Hounsfield unit (HU) value of the liver (CT value) not exceeding $40 \mathrm{HU}(7,8)$, or a spleen/liver HU ratio of more than 1.5 , determined by abdominal plain $\mathrm{CT}$ before, and three months after surgery. The median $\mathrm{HU}$ value of the liver and spleen parenchyma was calculated using a 3-point scale in the same abdominal CT slice.

Delayed gastric emptying (DGE) was classified into three grades (A, B and C) based on clinical impact, according to the definition proposed by the International Study Group of Pancreatic Surgery (ISGPS) (9).

Pancreatic fistula (PF) is characterized by amylase values in drained fluid greater than three times the upper limit of normal serum amylase values measured on POD 3. Three grades of PF (A, B, and C) were defined according to the standards set by the International Study Group of Pancreatic Fistula (10). Grade B and C patients were also evaluated in this series.

\section{Surgery}

PD was performed using the standard method. Anastomotic reconstruction was performed by the modified Child method described elsewhere (11). The pancreas division line was on the left side of the portal vein in all cases. SSPPD included the division of the stomach $2 \mathrm{~cm}$ proximal to the pyloric ring and the resection of the entire duodenum distal to the transection site, together with the gallbladder, distal 
common bile duct, and pancreatic head, as well as en bloc resection of the lymph nodes. If the tumour had invaded into the portal vein or superior mesenteric vein, the vein was resected and repaired to achieve curative surgery.

First, pancreaticojejunostomy (PJ) after SSPPD was performed by the Kakita method (12), using an external pancreatic duct stent (4-10 Fr polyethylene pancreatic drainage tube with a small knob: Sumitomo Bakelite Co., Japan) and duct-to-mucosa anastomosis. All stiches were done with monofilament non-absorbable suture (4-0 Prolene using the Kakita method and/or 5-0 or 6-0 Prolene for duct-to-mucosa sutures). Then, an end-toside choledochojejunostomy (CJ) with an internal stent (short stent: $3 \mathrm{~cm}$ in length, 4-10 Fr polyethylene pancreatic drainage tube with a small knob: Sumitomo Bakelite Co., Japan) was performed. After PJ and CJ, the gastrojejunostomy (GJ) was performed. A jejunal loop about $40 \mathrm{~cm}$ distal to the $\mathrm{CJ}$ was brought up anteriorly to the transverse colon and anastomosed to the stomach. The GJ was constructed by end-to-side twolayer Billroth-II (B-II) or Roux-en-Y (R-Y) reconstruction. After the procedure, duple drains were placed at the PJ, $\mathrm{CJ}$ and Winslow foramen.

\section{Statistical analysis}

Multivariate analysis was performed using variables that had been selected by univariate analysis on the basis of a cut-off probability value of $p<0.05$. The recommended cut-off values were defined by receiver operating characteristic (ROC) curve analysis. To determine the optimal cut-off points, the Youden index method was used $(13,14)$.
Differences between groups were analyzed using the chi-squared test. Odds ratios with $95 \%$ confidence interval $(95 \% \mathrm{Cl})$ were calculated using univariate or multivariate analysis. Statistical analyses were performed using the SPSS statistical software package, version 16.0 (SPSS Inc., Chicago, IL) at a significance level of $p<0.05$.

\section{RESULTS}

\section{Incidence of NAFLD}

The incidence of postoperative NAFLD was $27.0 \%$ (27/100). There were 25 patients whose CT values were less than $40 \mathrm{HU}$, while the remaining 2 patients had CT values exceeding $40 \mathrm{HU}$ but spleen/liver $\mathrm{HU}$ ratios exceeding 1.5. In 14 of these 27 patients, the spleen/liver HU ratios were more than 1.5 .

The pre- and postoperative liver CT values in the NA group were $52.8 \pm 8.25$ and $25.7 \pm 12.3$, respectively, showing a significant decrease in the postoperative value ( $p=0.0001)$. There were no significant differences in the pre- and postoperative spleen CT values between the two groups, and there was no difference in the liver $\mathrm{CT}$ value before and after surgery in the non-NA group (fig. 1).

\section{Risk factors for pre- and postoperative NAFLD}

Univariate analysis identified 7 factors that were associated with the development of NAFLD: age, R-Y reconstruction, serum Alb, AST, ALT, Fe and Zn levels at Preoperative, serum pre-alb, $\gamma$-GTP, Fe and $Z n$ levels at POD 7, serum AST, ALT, $\gamma$-GTP and Hb levels at POD 21, and serum AST, ALT and $y$-GTP levels at 3 months after

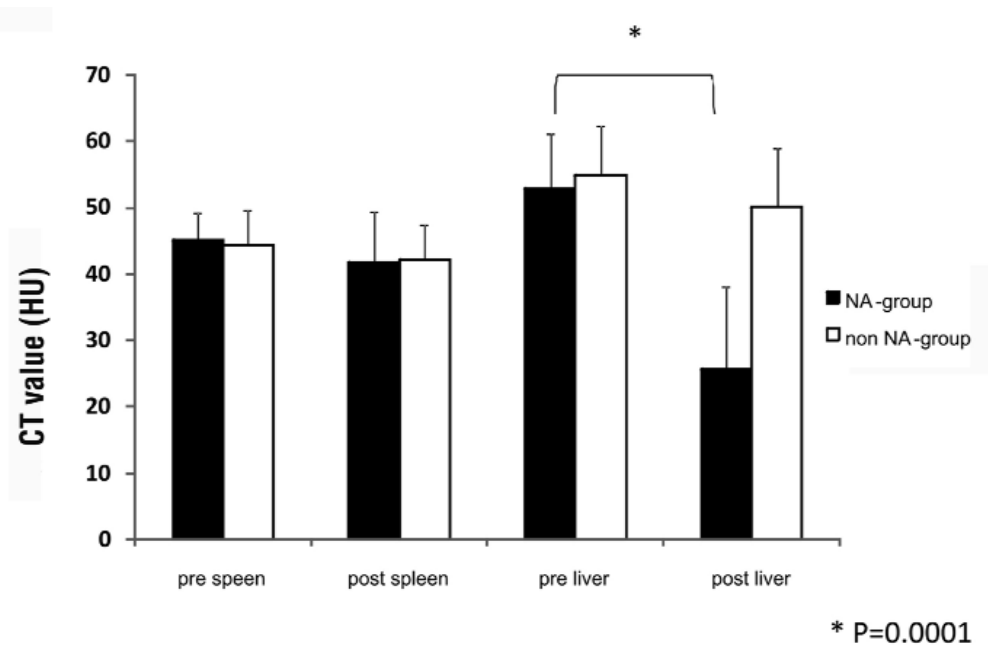

Figure 1 - Pre-and post-operative CT value in liver and spleen 
Table 1 - Univariate analysis of preoperative factors related to NAFLD after PD

\begin{tabular}{|c|c|c|c|c|c|}
\hline & NA group ( $n=27)$ & Non-NA group ( $\mathrm{n}=73$ ) & P-value & Odds ratio & 95\%C.I. \\
\hline Gender (M/F) & $14 / 13$ & $36 / 37$ & 0.150 & 1.935 & $0.778-4.750$ \\
\hline Age (yr) & $60.1(40.3-78.5)$ & $70.0(39.2-84.6)$ & 0.004 & 0.934 & $0.892-0.978$ \\
\hline Reconstruction (B-II/R-Y) & $8 / 19$ & $37 / 36$ & 0.043 & 0.378 & $0.147-0.971$ \\
\hline Bleeding (ml) & $600(280-2930)$ & $612.5(222-2911)$ & 0.938 & 1.000 & $0.147-0.971$ \\
\hline Op. time (hr:min) & $7: 55(5: 50-11: 49)$ & 8:02 (6:00-10:49) & 0.730 & 1.001 & $0.995-1.007$ \\
\hline Malignant disease (yes/no) & $25 / 2$ & $66 / 7$ & 0.749 & 1.306 & $0.254-6.714$ \\
\hline $\mathrm{BMI}$ & $21.6(12.5-31.1)$ & $21.1(16.9-31.1)$ & 0.596 & 0.972 & $0.876-1.079$ \\
\hline $\mathrm{T} . \mathrm{P}(\mathrm{g} / \mathrm{dl})$ & $6.6(5.7-7.8)$ & $6.6(4.7-8.6)$ & 0.603 & 1.200 & $0.603-2.387$ \\
\hline Alb $(\mathrm{g} / \mathrm{dl})$ & $3.6(2.4-4.3)$ & $3.3(2.2-4.5)$ & 0.080 & 2.216 & $0.909-5.402$ \\
\hline Pre alb (mg/dl) & $23.3(8.8-37.3)$ & $22.5(8.5-36.0)$ & 0.069 & 1.023 & $0.998-1.047$ \\
\hline $\mathrm{BS}(\mathrm{mg} / \mathrm{dl})$ & $100(50-191)$ & $94(60-294)$ & 0.445 & 1.004 & $0.993-1.015$ \\
\hline AST (IU/L) & $32(12-141)$ & $25(6-176)$ & 0.557 & 1.004 & $0.990-1.019$ \\
\hline $\mathrm{ALT}(\mathrm{IU} / \mathrm{L})$ & $43(10-325)$ & $36(3-231)$ & 0.075 & 1.007 & $0.999-1.015$ \\
\hline$\gamma$-GTP $(I U / L)$ & $142(17-898)$ & $82(10-768)$ & 0.100 & 1.002 & $1.000-1.005$ \\
\hline ALP $(I \mathrm{U} / \mathrm{L})$ & $367(149-1283)$ & $383(142-2038)$ & 0.936 & 1.000 & $0.949-1.001$ \\
\hline BUN (mg/dl) & $11(6-18)$ & $13(5-37)$ & 0.115 & 0.910 & $0.819-1.023$ \\
\hline $\mathrm{Cr}(\mathrm{mg} / \mathrm{dl})$ & $0.65(1.49)$ & $0.705(0.4-1.45)$ & 0.222 & 0.286 & $0.038-2.133$ \\
\hline $\mathrm{Na}(\mathrm{mEq} / \mathrm{l})$ & $141(134-147)$ & $140(131-147)$ & 0.310 & 1.080 & $0.931-1.253$ \\
\hline $\mathrm{K}(\mathrm{mEq} / \mathrm{l})$ & $4.2(3.6-5.2)$ & $4.2(2.6-5.4)$ & 0.244 & 1.735 & $0.687-4.382$ \\
\hline $\mathrm{Cl}(\mathrm{mEq} / \mathrm{l})$ & 105 (98-109) & $105(93-114)$ & 0.880 & 1.011 & $0.882-1.158$ \\
\hline WBC $\left(\times 10^{3} / \mu \mathrm{l}\right)$ & $5.0(2.1-8.4)$ & $6.05(2.1-5.5)$ & 0.027 & 1.000 & $0.999-1.000$ \\
\hline Neutro (\%) & $57.3(39.3-76.1)$ & $61.9(32.2-88.6)$ & 0.226 & 0.975 & $0.935-1.016$ \\
\hline $\mathrm{RBC}\left(\times 10^{6} / \mu \mathrm{l}\right)$ & $4.25(3.12-5.18)$ & $4.18(3.95-4.98)$ & 0.225 & 0.915 & $0.812-1.028$ \\
\hline $\mathrm{Hb}(\mathrm{g} / \mathrm{dl})$ & $12.3(8.3-17.0)$ & $12.3(8.1-15.9)$ & 0.258 & 1.145 & $0.906-1.448$ \\
\hline PIt $\left(x 10^{3} / \mu \mathrm{l}\right)$ & $21.9(13.2-32.7)$ & $21.0(8.8-68.7)$ & 0.716 & 0.954 & $0.894-1.018$ \\
\hline $\mathrm{CRP}(\mathrm{mg} / \mathrm{dl})$ & $0.1(0.1-2.84)$ & $0.16(0.1-13.8)$ & 0.195 & 0.496 & $0.172-1.432$ \\
\hline $\mathrm{Fe}(\mu \mathrm{g} / \mathrm{dl})$ & $85(26-176)$ & $63(16-146)$ & 0.006 & 1.024 & $1.007-1.042$ \\
\hline $\mathrm{Zn}(\mu \mathrm{g} / \mathrm{dl})$ & $86(53-130)$ & $71.5(40-114)$ & 0.011 & 1.037 & $1.009-1.067$ \\
\hline $\mathrm{Cu}(\mu \mathrm{g} / \mathrm{dl})$ & $105(79-160)$ & $101.5(17-154)$ & 0.479 & 1.007 & $0.988-1.025$ \\
\hline
\end{tabular}

Median (range), body mass index: BMI, total protein: TP, Albumin: Alb, blood sugar: BS, creatinine: Cr, hemoglobin: Hb, platelet: Plt, C-reactive protein: CRP, iron: $\mathrm{Fe}$, zinc: $\mathrm{Zn}$, copper: $\mathrm{Cu}$, postoperative days: POD

surgery (tables 1-4). As far as complications were concerned, PF and DGE were not associated risk factors for NAFLD (table 5).

After multivariate analysis was performed (table 6), only one factor remained significant. Fe $(p=0.034)$ was selected as the most potent preoperative risk factor for NAFLD.

For Fe at Pre, ROC curve analysis revealed that 73.5 $\mu \mathrm{g} / \mathrm{dl}$ was an appropriate cut-off value for detection of NAFLD preoperatively (sensitivity: $64.0 \%$, specificity: $70.5 \%)$.

Most prominent point disclosed two parameters such as sensitivity (0.640) and 1-specificity (0.705) (fig. 2).

\section{DISCUSSION}

NAFLD includes a spectrum of hepatic histological features ranging from hepatic steatosis to inflammatory changes with or without fibrosis, referred to as nonalcoholic steatohepatitis (NASH). NAFLD is particularly common among overweight and obese individuals (15, 16). NASH may progress to advanced fibrosis and cirrhosis in a significant proportion of those affected (17). However, its exact prevalence and pathophysiology are still unclear. Several factors including oxidative stress, iron overload, and altered lipid metabolism are reportedly associated with the progression of simple steatosis to $\operatorname{NASH}(5,18)$. Overall, a strong association has been observed between NAFLD and insulin resistance, leading to the suggestion that NAFLD may be the hepatic manifestation of metabolic syndrome (19). Several factors, such as liver injury mediated by endotoxins or cytokines and oxidative stress, have been postulated. These are sometimes induced by major 
Table 2 - Univariate analysis of factors related to NAFLD after PD at POD 7

\begin{tabular}{|c|c|c|c|c|c|}
\hline & NA group ( $n=27$ ) & Non-NA group $(n=73)$ & P-value & Odds ratio & 95\%C.I. \\
\hline$\overline{\mathrm{BMI}}$ & $20.2(13.0-31.8)$ & $20.0(15.9-29.1)$ & 0.419 & 0.974 & 0.913-1.038 \\
\hline $\mathrm{T} . \mathrm{P}(\mathrm{g} / \mathrm{dl})$ & $5.8(4.0-7.2)$ & $6.0(4.2-7.9)$ & 0.169 & 0.666 & $0.374-1.188$ \\
\hline Alb (g/dl) & $2.5(1.9-3.4)$ & $2.6(1.6-3.6)$ & 0.738 & 1.214 & $0.389-3.788$ \\
\hline Pre alb (mg/dl) & $11.8(5.9-27.8)$ & $11.4(3.5-28.5)$ & 0.468 & 1.034 & $0.945-1.132$ \\
\hline $\mathrm{BS}(\mathrm{mg} / \mathrm{dl})$ & $98(61-139)$ & $103.5(61-373)$ & 0.097 & 0.986 & $0.969-1.003$ \\
\hline AST (IU/L) & $29(10-122)$ & $23(8-101)$ & 0.145 & 1.017 & $0.994-1.041$ \\
\hline $\mathrm{ALT}(\mathrm{I} \mathrm{I} / \mathrm{L})$ & $31(10-155)$ & $26(7-320)$ & 0.388 & 1.004 & $0.995-1.014$ \\
\hline$\gamma$-GTP $(I \mathrm{I} / \mathrm{L})$ & $52(22-262)$ & $42(10-187)$ & 0.158 & 1.007 & $0.997-1.016$ \\
\hline $\mathrm{A}[\mathrm{P}(\mathrm{I} \mathrm{U} / \mathrm{L})$ & $464(110-948)$ & $333(172-1369)$ & 0.395 & 1.001 & $0.999-1.002$ \\
\hline BUNN (mg/dl) & $9.5(4-29)$ & $11(5-44)$ & 0.187 & 0.940 & $0.857-1.031$ \\
\hline $\mathrm{Cr}(\mathrm{mg} / \mathrm{dl})$ & $0.67(0.33-1.16)$ & $0.66(0.28-1.43)$ & 0.361 & 0.931 & $0.971-2.618$ \\
\hline $\mathrm{Na}(\mathrm{mEq} / \mathrm{l})$ & $138(130-145)$ & $136(128-151)$ & 0.257 & 1.068 & $0.953-1.197$ \\
\hline $\mathrm{K}(\mathrm{mEq} / \mathrm{l})$ & $4.4(3.5-4.9)$ & $4.4(3.2-5.6)$ & 0.687 & 0.964 & $0.805-1.153$ \\
\hline $\mathrm{Cl}(\mathrm{mEq} / \mathrm{l})$ & $99(93-105)$ & $99(89-110)$ & 0.567 & 1.035 & $0.921-1.162$ \\
\hline $\mathrm{WBC}\left(\times 10^{3} / \mu \mathrm{l}\right)$ & $8.8(3.0-12.6)$ & $9.35(3.2-19.9)$ & 0.048 & 1.000 & $1.000-1.000$ \\
\hline Neutro (\%) & $76.3(55.3-87.6)$ & $76.4(53.4-92.7)$ & 0.284 & 0.968 & $0.912-1.027$ \\
\hline $\mathrm{RBC}\left(\times 10^{6} / \mu\right)$ & $4.06(3.11-4.49)$ & $3.97(2.98-4.38)$ & 0.568 & 1.036 & $0.921-1.089$ \\
\hline $\mathrm{Hb}(\mathrm{g} / \mathrm{dl})$ & $10.8(8.1-15.8)$ & $10.9(7.4-14.5)$ & 0.753 & 1.046 & $0.792-1.381$ \\
\hline Plt $\left(x 10^{3} / \mu l\right)$ & $23.5(11.7-40.2)$ & $24.8(10.6-56.0)$ & 0.154 & 0.954 & $0.894-1.018$ \\
\hline $\mathrm{CRP}(\mathrm{mg} / \mathrm{dl})$ & $2.18(0.1-13.95)$ & $4.7(0.18-20.72)$ & 0.263 & 0.932 & $0.837-1.038$ \\
\hline $\mathrm{Fe}(\mu \mathrm{g} / \mathrm{dl})$ & $29(18-148)$ & $25(11-82)$ & 0.012 & 1.054 & $1.012-1.099$ \\
\hline $\mathrm{Zn}(\mu \mathrm{g} / \mathrm{dl})$ & $74(43-118)$ & $67.5(36-100)$ & 0.036 & 1.033 & $1.002-1.065$ \\
\hline $\mathrm{Cu}(\mu \mathrm{g} / \mathrm{dl})$ & $87(57-126)$ & $92(30-143)$ & 0.510 & 0.992 & $0.970-1.015$ \\
\hline
\end{tabular}

Median (range), body mass index: BMI, total protein: TP, Albumin: Alb, blood sugar: BS, creatinine: Cr, hemoglobin: Hb, platelet: Plt, C-reactive protein: CRP, iron: Fe, zinc: Zn, copper: Cu, postoperative days: POD

abdominal surgery. A previous report has documented that NAFLD was observed in $37 \%$ of the patients who underwent PD, and that the risk factors for NAFLD after $\mathrm{PD}$ were pancreatic cancer, the pancreatic resection line and postoperative diarrhoea (3). Another study found that $23 \%$ of patients developed NAFLD after PD, and that the only evident associated risk factor was pancreatic cancer (4). All of these risk factors become clear after the operation.

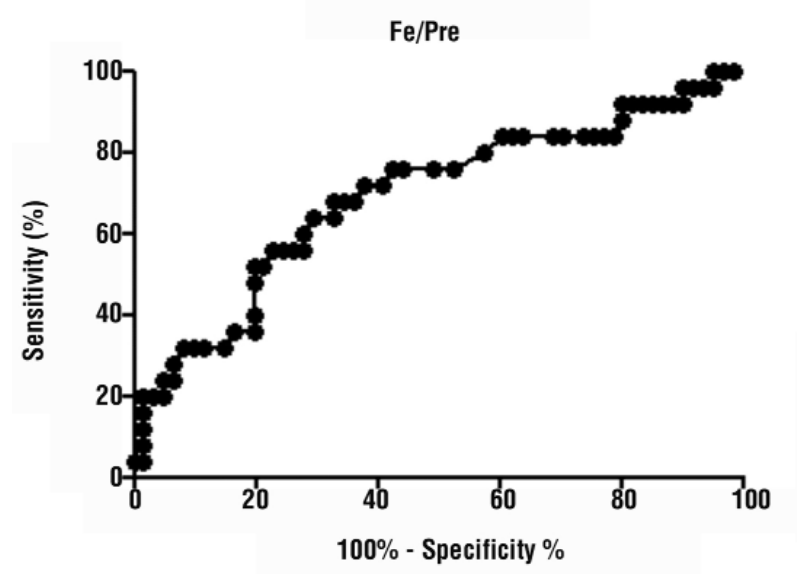

Fe $73.5 \mu \mathrm{g} / \mathrm{dl}$

Sensitivity $64 \%$

Specificity $70.5 \%$
In the present study, for the first time, we investigated the pre- and postoperative risk factors for NAFLD after PD, and found that the preoperative serum level of Fe was significantly associated with this complication. In our series, NAFLD developed in $27 \%$ of patients who underwent PD, being a proportion which is comparable with a previously reported figure (3). In the present NA group, there were significant differences in the liver $\mathrm{CT}$ values at Pre and 3 months after PD, suggesting that $C T$ 
Table 3 - Univariate analysis of factors related to NAFLD after PD at POD 21

\begin{tabular}{|c|c|c|c|c|c|}
\hline & NA group ( $n=27$ ) & Non-NA group $(n=73)$ & P-value & Odds ratio & 95\%C.I. \\
\hline$\overline{\mathrm{BMI}}$ & $21.1(12.9-32.4)$ & $20.6(16.6-30.5)$ & 0.983 & 0.999 & $0.940-1.063$ \\
\hline $\mathrm{T} . \mathrm{P}(\mathrm{g} / \mathrm{dl})$ & $5.1(4.4-6.1)$ & $5.2(4.0-6.6)$ & 0.112 & 0.489 & $0.203-1.181$ \\
\hline Alb (g/dl) & $2.2(1.7-3.0)$ & $2.2(1.6-3.5)$ & 0.359 & 1.211 & $0.804-1.824$ \\
\hline Pre alb (mg/dl) & $12.7(6.7-19.4)$ & $10.9(4.0-23.5)$ & 0.074 & 1.123 & $0.989-1.275$ \\
\hline $\mathrm{BS}(\mathrm{mg} / \mathrm{dl})$ & $149(86-265)$ & $128(62-332)$ & 0.769 & 0.999 & $0.991-1.007$ \\
\hline AST (IU/L) & $36(15-174)$ & $36(11-185)$ & 0.721 & 1.002 & $0.991-1.014$ \\
\hline $\mathrm{ALT}(\mathrm{I} \mathrm{I} / \mathrm{L})$ & $72(17-371)$ & $65(10-317)$ & 0.259 & 1.003 & $0.997-1.010$ \\
\hline$\gamma$-GTP $(I \mathrm{I} / \mathrm{L})$ & $128(28-320)$ & $96(13-472)$ & 0.238 & 1.003 & $0.998-1.008$ \\
\hline ALP $(I \mathrm{I} / \mathrm{L})$ & $445(154-1361)$ & $387(147-2361)$ & 0.991 & 1.000 & $0.999-1.001$ \\
\hline BUNN (mg/dl) & $12(5-31)$ & $12(6-38)$ & 0.412 & 0.996 & $0.891-1.049$ \\
\hline $\mathrm{Cr}(\mathrm{mg} / \mathrm{dl})$ & $0.58(0.28-2.18)$ & $0.61(0.32-2.18)$ & 0.262 & 0.285 & $0.032-2.553$ \\
\hline $\mathrm{Na}(\mathrm{mEq} / \mathrm{l})$ & $136(126-143)$ & $135(123-143)$ & 0.271 & 1.076 & $0.945-1.225$ \\
\hline $\mathrm{K}(\mathrm{mEq} / \mathrm{l})$ & $4.4(3.6-5.4)$ & $4.2(2.5-6)$ & 0.109 & 1.855 & $0.871-3.947$ \\
\hline $\mathrm{Cl}(\mathrm{mEq} / \mathrm{l})$ & $102(91-109)$ & $101(92-110)$ & 0.264 & 0.968 & $0.915-1.025$ \\
\hline $\mathrm{WBC}\left(\times 10^{3} / \mu \mathrm{l}\right)$ & $5.8(3.1-11.1)$ & $6.9(3.0-24.5)$ & 0.112 & 1.000 & $1.000-1.000$ \\
\hline Neutro (\%) & $63.4(47.8-80.7)$ & $68.1(35.0-94.8)$ & 0.278 & 0.980 & $0.944-1.017$ \\
\hline $\mathrm{RBC}\left(\times 10^{6} / \mu \mathrm{l}\right)$ & $4.18(3.10-4.38)$ & $4.02(2.81-4.32)$ & 0.120 & 1.015 & $1.014-1.181$ \\
\hline $\mathrm{Hb}(\mathrm{g} / \mathrm{dl})$ & $11.5(7.8-14.6)$ & $10.5(7.4-14.9)$ & 0.041 & 1.355 & $1.012-1.814$ \\
\hline Plt $\left(x 10^{3} / \mu l\right)$ & $29.2(15.4-51.8)$ & $30.8(10.1-69.3)$ & 0.665 & 0.995 & $0.944-1.037$ \\
\hline $\mathrm{CRP}(\mathrm{mg} / \mathrm{dl})$ & $0.89(0.1-13.95)$ & $1.22(0.1-11.66)$ & 0.581 & 0.951 & $0.797-1.135$ \\
\hline $\mathrm{Fe}(\mu \mathrm{g} / \mathrm{dl})$ & $29(8-87)$ & $28.5(10-106)$ & 0.415 & 1.011 & $0.985-1.038$ \\
\hline $\mathrm{Zn}(\mu \mathrm{g} / \mathrm{dl})$ & $91.5(72-126)$ & $97(39-133)$ & 0.451 & 1.011 & $0.983-1.040$ \\
\hline $\mathrm{Cu}(\mu \mathrm{g} / \mathrm{dl})$ & $100(46-131)$ & $104(17-180)$ & 0.741 & 0.997 & $0.977-1.016$ \\
\hline
\end{tabular}

Median (range), body mass index: BMI, total protein: TP, Albumin: Alb, blood sugar: BS, creatinine: Cr, haemoglobin: Hb, platelet: Plt, C-reactive protein: CRP, iron: Fe, zinc: Zn, copper: Cu, postoperative days: POD

Table 4 - Univariate analysis of factors related to NAFLD after PD at 3 months after surgery

\begin{tabular}{|c|c|c|c|c|c|}
\hline & NA group ( $n=27$ ) & Non-NA group $(\mathrm{n}=73)$ & P-value & Odds ratio & 95\%C.I. \\
\hline BMI & $19.7(13-30.9)$ & $18.9(15-27.6)$ & 0.890 & 0.995 & $0.926-1.069$ \\
\hline T.P $(\mathrm{g} / \mathrm{dl})$ & $6.3(4.9-7.3)$ & $6.5(3.6-7.9)$ & 0.786 & 0.923 & $0.519-1.643$ \\
\hline Alib $(g / d l)$ & $3.1(2.1-4.4)$ & $3.0(1.6-4.3)$ & 0.405 & 1.416 & $0.624-3.213$ \\
\hline Pre alb (mg/dl) & $13.6(5.7-26.4)$ & $13.4(3.8-29.9)$ & 0.778 & 0.987 & $0.896-1.087$ \\
\hline $\mathrm{BS}(\mathrm{mg} / \mathrm{dl})$ & $105(73-371)$ & $111(50-421)$ & 0.297 & 0.996 & $0.988-1.004$ \\
\hline AST (IU/L) & $33(11-110)$ & $23(6-85)$ & 0.018 & 1.033 & $1.006-1.062$ \\
\hline ALT $(I U / L)$ & $36(12-127)$ & $19(12-214)$ & 0.102 & 1.014 & $0.997-1.031$ \\
\hline$\gamma$-GTP $(I \mathrm{I} / L)$ & $36(14-243)$ & $26(10-94)$ & 0.121 & 1.018 & $0.995-1.040$ \\
\hline ALP $(I \mathrm{I} / \mathrm{L})$ & $377(139-872)$ & $292(169-880)$ & 0.058 & 1.004 & $1.000-1.008$ \\
\hline BUUN (mg/dl) & $11(7-20)$ & $12(5-62)$ & 0.111 & 0.903 & $0.797-1.024$ \\
\hline $\mathrm{Cr}(\mathrm{mg} / \mathrm{dl})$ & $0.63(0.31-1.1)$ & $0.63(0.31-1.62)$ & 0.287 & 0.268 & $0.224-3.021$ \\
\hline $\mathrm{Na}(\mathrm{mEq} / \mathrm{l})$ & $141(134-144)$ & $139(127-144)$ & 0.023 & 1.274 & $1.033-1.571$ \\
\hline $\mathrm{K}(\mathrm{mEq} / \mathrm{l})$ & $4.3(3.2-5.5)$ & $4.3(2.8-5.8)$ & 0.888 & 1.060 & $0.471-2.387$ \\
\hline $\mathrm{Cl}(\mathrm{mEq} / \mathrm{l})$ & $105(96-116)$ & $104(90-113)$ & 0.155 & 1.091 & $0.968-1.229$ \\
\hline WBC $\left(\times 10^{3} / \mu \mathrm{l}\right)$ & $5.3(2.1-11.1)$ & $4.9(1.5-19.2)$ & 0.629 & 1.000 & $1.000-1.000$ \\
\hline Neutro $(\%)$ & $59.7(29.9-93.6)$ & $56.1(24.4-92.6)$ & 0.205 & 1.021 & $0.989-1.054$ \\
\hline $\mathrm{RBC}\left(\times 10^{6} / \mu \mathrm{l}\right)$ & $4.20(4.10-4.67)$ & $3.98(2.87-4.76)$ & 0.564 & 1.010 & $0.814-1.168$ \\
\hline $\mathrm{Hb}(\mathrm{g} / \mathrm{dl})$ & $11.0(8.3-13.4)$ & $11.0(6.6-14.8)$ & 0.465 & 1.133 & $0.811-1.582$ \\
\hline PIt $\left(\times 10^{3} / \mu l\right)$ & $24.5(12.2-52.0)$ & $20.5(3.5-61.3)$ & 0.239 & 1.030 & $0.981-1.082$ \\
\hline $\mathrm{CRP}(\mathrm{mg} / \mathrm{dl})$ & $0.16(0.1-12.21)$ & $0.25(0.1-11.66)$ & 0.491 & 1.066 & $0.888-1.281$ \\
\hline $\mathrm{Fe}(\mu \mathrm{g} / \mathrm{dl})$ & $57(26-124)$ & $54(14-114)$ & 0.381 & 1.014 & $0.983-1.046$ \\
\hline $\mathrm{Zn}(\mu \mathrm{g} / \mathrm{dl})$ & $73.5(35-125)$ & $68(29-128)$ & 0.501 & 1.008 & $0.985-1.030$ \\
\hline $\mathrm{Cu}(\mu \mathrm{g} / \mathrm{dl})$ & $86.5(51-129)$ & $86.0(17-140)$ & 0.846 & 0.998 & $0.976-1.020$ \\
\hline
\end{tabular}

Median (range), body mass index: BMI, total protein: TP, Albumin: Alb, blood sugar: BS, creatinine: $\mathrm{Cr}$, haemoglobin: Hb, platelet: Plt, C-reactive protein: CRP, iron: $\mathrm{Fe}$, zinc: Zn, copper: $\mathrm{Cu}$, postoperative days: POD 
Table 5 - Univariate analysis of postoperative complications related to NAFLD after PD

\begin{tabular}{lccccc}
\hline & NA group $(\mathbf{n = 2 7})$ & Non-NA group $(\mathbf{n = 7 3})$ & P-value & Odds ratio & 95\%C.I. \\
\hline $\mathrm{PF}($ yes/no) & $6 / 21$ & $17 / 56$ & 0.632 & 0.762 & $0.251-2.317$ \\
\hline $\mathrm{DGE}(\mathrm{yes} / \mathrm{no})$ & $4 / 23$ & $17 / 56$ & 0.375 & 0.583 & $0.177-1.921$ \\
\hline
\end{tabular}

Pancreatic fistula: PF, Delayed gastric emptying: DGE

Table 6 - Multivariate analysis of selected clinical risk factors for NAFLD after PD

\begin{tabular}{lccc}
\hline & P-value & Odds ratio & 95\%C.I. \\
\hline age & 0.061 & 0.923 & $0.849-1.004$ \\
R-Y reconstruction & 0.223 & 0.359 & $0.069-1.863$ \\
\hline AST after 3 months & 0.027 & 1.053 & $1.006-1.103$ \\
\hline Hb at 21 POD & 0.115 & 1.225 & $0.952-1.578$ \\
\hdashline Preop.Fe & 0.034 & 1.052 & $1.007-1.041$ \\
\hline Preop.Zn & 0.030 & 1.062 & $1.006-1.121$ \\
Fe at 7 POD & 0.026 & 1.100 & $1.012-1.197$ \\
\hline Zn at 7 POD & 0.223 & 0.966 & $0.914-1.021$ \\
\hline
\end{tabular}

Hemoglobin: $\mathrm{Hb}$, iron: $\mathrm{Fe}$, zinc: $\mathrm{Zn}$, copper: $\mathrm{Cu}$, preoperation: preope, postoperative days: POD

at 3 months after PD is advisable to screen for NAFLD. The preoperative AST and ALT levels in the NA group were significantly higher compared to the non NA group. This result would suggest that preoperative liver function seems to become a risk factor for NAFLD post PD. However, no significant difference was observed at multivariate analysis. As a matter of fact, multivariate analysis revealed that the preoperative serum Fe level, was the only significant risk factor for NAFLD. Fe has also been extensively investigated in relation to liver injury $(19,20)$. Bonkovsky et al. reported that the prevalence of hemochromatosis protein gene (HFE) mutations associated with hereditary hemochromatosis was higher among patients with NASH and NAFLD (5). A significantly higher prevalence of HFE mutations in NAFLD patients has been reported to be a factor responsible for liver fibrosis by increasing hepatic iron deposition (21), but recent studies have failed to confirm this (22). Kosuge et al. compared the level of Fe with those of CRP, IL- 6 and hepcidin in PD patients before and after surgery, and found that Fe was dependent on the expression of the latter three factors. Surgical stress induces inflammation, as expressed by an increase of CRP and IL-6, resulting in an increase of hepcidin production, which in turn suppresses the release of iron from macrophages at POD 3. The Fe level is suppressed thereafter (23).

In a clinical setting, it would be useful to predict the development of postoperative NAFLD before surgery.
Table 7- Analysis of patients in the NA and non-NA groups using selected cut-off values

\begin{tabular}{lccc}
\hline & $\begin{array}{c}\text { NA group } \\
(\mathbf{n}=\mathbf{2 7})\end{array}$ & $\begin{array}{c}\text { Non-NA group } \\
(\mathbf{n}=63)\end{array}$ & P-value \\
\hline $\mathrm{Fe}(\geq 73.5 \mu \mathrm{g} / \mathrm{dl}$ & & & \\
$/<73.5 \mu \mathrm{g} / \mathrm{dl}$ & $21 / 6$ & $16 / 57$ & 0.0001 \\
\hdashline $\mathrm{Zn}(\geq 78.5 \mu \mathrm{g} / \mathrm{dl}$ & & & \\
$/<78.5 \mu \mathrm{g} / \mathrm{dl}$ & $16 / 11$ & $24 / 49$ & 0.016 \\
\hline
\end{tabular}

For this purpose, the preoperative cut-off values of Fe postulated in the present study would be helpful. Preoperative $\mathrm{Fe}$ values of $73.5 \mu \mathrm{g} / \mathrm{dl}$, efficiently discriminated patients who would develop NAFLD after PD from those who wouldn't. Patients who have a Fe level above this cut-off values should be recognized as a high-risk group for NAFLD after PD.

One interesting question is whether or not postoperative NAFLD could be avoided by reduction of the preoperative Fe level. To answer this question, a prospective trial of aggressive preoperative control of $\mathrm{Fe}$ will be necessary.

\section{CONCLUSION}

NAFLD after PD was observed in $27 \%$ of the patients in our study. Patients who have preoperative Fe value exceeding $73.5 \mu \mathrm{g} / \mathrm{dl}$ should be carefully monitored for the development of NAFLD after PD.

\section{Conflict of interests}

The authors declare no conflict of interests.

\section{REFERENCES}

1. Horstmann O, Markus PM, Ghadimi MB, Becker H. Pylorus preservation has no impact on delayed gastric emptying after pancreatic head resection. Pancreas. 2004 Jan;28(1):69-74. PubMed PMID: 14707733. Epub 2004/01/07. eng.

2. Yeo CJ, Cameron JL, Sohn TA, Lillemoe KD, Pitt HA, Talamini MA, et al. Six hundred fifty consecutive pancreaticoduodenectomies in the 1990s: pathology, complications, and outcomes. Ann Surg. 1997 Sep;226(3):248-57; discussion 57-60. PubMed PMID: 9339931. Pubmed Central PMCID: 1191017. Epub 1997/10/27. eng.

3. Kato H, Isaji S, Azumi Y, Kishiwada M, Hamada T, Mizuno S, et al. Development of nonalcoholic fatty liver disease (NAFLD) and nonalcoholic steatohepatitis (NASH) after pancreaticoduodenectomy: proposal of a postoperative NAFLD scoring system. J Hepatobiliary 
Pancreat Sci. 2010 May;17(3):296-304. PubMed PMID: 19809782. Epub 2009/10/08. eng.

4. Tanaka N, Horiuchi A, Yokoyama T, Kaneko G, Horigome N, Yamaura T, et al. Clinical characteristics of de novo nonalcoholic fatty liver disease following pancreaticoduodenectomy. J Gastroenterol. 2011 Jun;46(6):758-68. PubMed PMID: 21267748. Epub 2011/01/27. eng.

5. Bonkovsky HL, Jawaid Q, Tortorelli K, LeClair P, Cobb J, Lambrecht RW, et al. Non-alcoholic steatohepatitis and iron: increased prevalence of mutations of the HFE gene in non-alcoholic steatohepatitis. J Hepatol. 1999 Sep;31(3):421-9. PubMed PMID: 10488699. Epub 1999/09/17. eng.

6. Schnabl B, Czech B, Valletta D, Weiss TS, Kirovski G, Hellerbrand C. Increased expression of Zinc finger protein 267 in non-alcoholic fatty liver disease. Int J Clin Exp Pathol. 2011;4(7):661-6. PubMed PMID: 22076166. Pubmed Central PMCID: 3209606 . Epub 2011/11/15. eng.

7. Pamilo M, Sotaniemi EA, Suramo I, Lahde S, Arranto AJ. Evaluation of liver steatotic and fibrous content by computerized tomography and ultrasound. Scand J Gastroenterol. 1983 Sep;18(6):743-7. PubMed PMID: 6669938. Epub 1983/09/01. eng.

8. Hamer OW, Aguirre DA, Casola G, Sirlin CB. Imaging features of perivascular fatty infiltration of the liver: initial observations. Radiology. 2005 Oct;237(1):159-69. PubMed PMID: 16100085. Epub 2005/08/16. eng.

9. Wente MN, Bassi C, Dervenis C, Fingerhut A, Gouma DJ, Izbicki JR, et al. Delayed gastric emptying (DGE) after pancreatic surgery: a suggested definition by the International Study Group of Pancreatic Surgery (ISGPS). Surgery. 2007 Nov;142(5):761-8. PubMed PMID: 17981197. Epub 2007/11/06. eng.

10. Bassi C, Dervenis C, Butturini G, Fingerhut A, Yeo C, Izbicki J, et al. Postoperative pancreatic fistula: an international study group (ISGPF) definition. Surgery. 2005 Jul;138(1):8-13. PubMed PMID: 16003309. Epub 2005/07/09. eng.

11. Tani M, Terasawa H, Kawai M, Ina S, Hirono S, Uchiyama K, et al. Improvement of delayed gastric emptying in pylorus-preserving pancreaticoduodenectomy: results of a prospective, randomized, controlled trial. Ann Surg. 2006 Mar;243(3):316-20. PubMed PMID: 16495694. Pubmed Central PMCID: 1448934. Epub 2006/02/24. eng.

12. Kakita A, Takahashi T, Yoshida M, Furuta K. A simpler and more reliable technique of pancreatojejunal anastomosis. Surg Today. 1996;26(7):532-5. PubMed PMID: 8840437. Epub 1996/01/01. eng.

13. Perkins NJ, Schisterman EF. The inconsistency of "optimal" cutpoints obtained using two criteria based on the receiver operating characteristic curve. Am J Epidemiol. 2006 Apr 1;163(7):670-5. PubMed PMID: 16410346. Pubmed Central PMCID: 1444894. Epub 2006/01/18. eng.

14. Akobeng AK. Understanding diagnostic tests 1 : sensitivity, specificity and predictive values. Acta Paediatr. 2007 Mar;96(3):338-41. PubMed PMID: 17407452. Epub 2007/04/05. eng.

15. Youssef WI, McCullough AJ. Steatohepatitis in obese individuals. Best Pract Res Clin Gastroenterol. 2002 0ct;16(5):733-47. PubMed PMID: 12406442. Epub 2002/10/31. eng.

16. Bugianesi E, Marzocchi R, Villanova N, Marchesini G. Non-alcoholic fatty liver disease/non-alcoholic steatohepatitis (NAFLD/NASH): treatment. Best Pract Res Clin Gastroenterol. 2004 Dec;18(6):110516. PubMed PMID: 15561641. Epub 2004/11/25. eng.

17. Harrison SA, Torgerson S, Hayashi PH. The natural history of nonalcoholic fatty liver disease: a clinical histopathological study. Am J Gastroenterol. 2003 Sep;98(9):2042-7. PubMed PMID: 14499785. Epub 2003/09/23. eng.

18. de Almeida IT, Cortez-Pinto H, Fidalgo G, Rodrigues D, Camilo ME. Plasma total and free fatty acids composition in human non-alcoholic steatohepatitis. Clin Nutr. 2002 Jun;21(3):219-23. PubMed PMID: 12127930. Epub 2002/07/20. eng.

19. Marchesini G, Bugianesi E, Forlani G, Cerrelli F, Lenzi M, Manini R, et al. Nonalcoholic fatty liver, steatohepatitis, and the metabolic syndrome. Hepatology. 2003 Apr;37(4):917-23. PubMed PMID: 12668987. Epub 2003/04/02. eng.

20. Nelson JE, Klintworth H, Kowdley KV. Iron Metabolism in Nonalcoholic Fatty Liver Disease. Curr Gastroenterol Rep. 2011 Nov 29. PubMed PMID: 22124850. Epub 2011/11/30. Eng.

21. Chitturi S, Weltman M, Farrell GC, McDonald D, Kench J, Liddle C, et al. HFE mutations, hepatic iron, and fibrosis: ethnic-specific association of NASH with C282Y but not with fibrotic severity. Hepatology. 2002 Jul;36(1):142-9. PubMed PMID: 12085358. Epub 2002/06/27. eng.

22. Yamauchi N, Itoh Y, Tanaka Y, Mizokami M, Minami M, Morita A, et al. Clinical characteristics and prevalence of GB virus C, SEN virus, and HFE gene mutation in Japanese patients with nonalcoholic steatohepatitis. J Gastroenterol. 2004 Jul;39(7):654-60. PubMed PMID: 15293136. Epub 2004/08/05. eng.

23. Kosuge T, Sawada T, Shimoda M, Kita J, Tomosugi N, Kubota K. Increased hepcidin production impairs iron metabolism after pancreatoduodenectomy. World J Surg. 2010 0ct;34(10):2452-6. PubMed PMID: 20517606. Epub 2010/06/03. eng. 